\title{
A Generative Simulation Platform for Multi-agent Systems with Incentives
}

\author{
Zhengwei $\mathrm{Wu}^{*}$ \\ University of California, Santa \\ Barbara \\ zhengwei@ucsb.edu \\ Xinlei Chen \\ Carnegie Mellon University \\ xinlei.chen@sv.cmu.edu
}

\author{
Xiaoxi Zhang* \\ Carnegie Mellon University \\ xiaoxiz2@andrew.cmu.edu \\ Pei Zhang \\ Carnegie Mellon University \\ peizhang@cmu.edu \\ Carlee Joe-Wong \\ Carnegie Mellon University \\ cjoewong@andrew.cmu.edu
}

\author{
Susu $\mathrm{Xu}^{*}$ \\ Carnegie Mellon University \\ ssxthu@gmail.com
}

\author{
Hae Young Noh \\ Stanford University \\ noh@stanford.edu
}

\begin{abstract}
Multi-agent systems have attracted much attention in the recent years due to their capabilities to handle complex and computation-heavy tasks and compatibility with incentive schemes. Considering the difficulty of creating an actual prototype and environment for evaluation, a simulation platform is a cheap and efficient way in analyzing and testing, prior to real environmental implementations. Existing simulators for multi-agent systems are inadequate to analyze the effects of different customized incentive schemes on agents' behavior patterns due to two reasons: 1) They lack the functionality to support various types of complex incentives, e.g., mixture of monetary incentives and non-monetary incentives, which influences agents' behaviors explicitly and implicitly; 2) They are not able to emulate heterogeneous agents' realtime behaviors that are influenced by complex incentives and deviate from their original behavior patterns shown in historical traces. In this paper, we focus on mobile agents that can move in a patio-temporal space, and we present a physical knowledge aided multi-agent simulation platform considering the influence of both direct and indirect incentives unified through a general utility-driven agent reaction function. The behaviors of agents are then emulated in three

${ }^{\bar{*}}$ These three authors contribute equally to this work. Xinlei Chen is corresponding author.
\end{abstract}

\section{(c) (†)}

This work is licensed under a Creative Commons Attribution International 4.0 License. UbiComp/ISWC '20 Adjunct, September 12-16, 2020, Virtual Event, Mexico (c) 2020 Copyright held by the owner/author(s).

ACM ISBN 978-1-4503-8076-8/20/09.

https://doi.org/10.1145/3410530.3414590 behavioral models: myopic, semi-myopic, and farsighted, by varying the assumption of agents in maximizing their utilities and integrating the physical knowledge and historical mobility patterns. We finally examine the effectiveness of the platform in incentivizing vehicle agents to optimize the final distribution of the agents through a ride-sharing vehicle experimental scenario. The emulated agents' behaviors can also be collected into data traces for analyzing other patterns of the agents.

\section{ACM Reference Format:}

Zhengwei Wu, Xiaoxi Zhang, Susu Xu, Xinlei Chen, Pei Zhang, Hae Young Noh, and Carlee Joe-Wong. 2020. A Generative Simulation Platform for Multi-agent Systems with Incentives. In Adjunct Proceedings of the 2020 ACM International foint Conference on Pervasive and Ubiquitous Computing and Proceedings of the 2020 ACM International Symposium on Wearable Computers (UbiComp/ISWC '20 Adjunct), September 12-16, 2020, Virtual Event, Mexico. ACM, New York, NY, USA, 8 pages. https://doi.org/10.1145/3410530.3414590

\section{INTRODUCTION}

\section{Multi-agent System}

Multi-agent systems have drawn great attention recently due to their capabilities to handle complex and computation heavy tasks [1-4]. Benefiting from the distributed computation, the tasks can be distributed to multiple agents to execute independently or collaboratively, which have been proved effective in various applications including environmental monitoring, urban surveillance, system diagnosis, market simulation and etc [5-7].

Multi-agent systems consist of multiple agents with local goals that cooperate with each other to reach a global object. Different from monolithic systems, which are restrained to solve simple node interactions, multi-agent systems are able to deal with complicated interactions between multiple nodes in distributed ways. In addition, multi-agent systems can take 


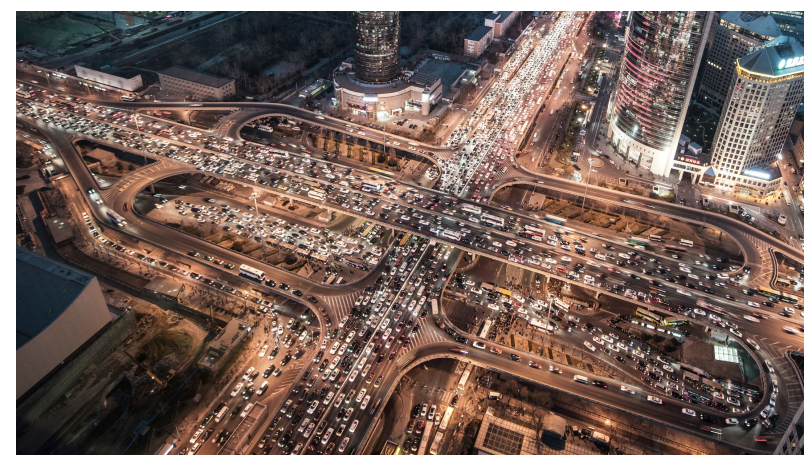

Figure 1: Example of multi-agent in a city

full advantage of AI technology within their specific agent strategies to further expand the list of applications. As a result, technologies implemented with multi-agent systems have successfully solved real-world industrial problems.

\section{Incentive Schemes for Multi-agent Systems}

Incentives are crucial for a multi-agent system, since they affect the strategy and behavior of each agent, and finally determine the performance of the entire system [8-11]. Agents' behaviors are usually influenced by two kinds of incentives: direct and indirect incentives. The direct incentives refer to monetary bonuses that agents can accept by taking certain actions. The indirect incentives refer to non-monetary factors that are able to influence decisions of individual agents, which can usually be extracted from the real-world dynamics of the environment.

The direct incentives, in the form of monetary bonuses, are intuitive attempts to change the agents' behaviors. The direct incentives are usually manually created and easy to control. The major question is how to ensure effectiveness of incentivizing agents with minimum cost.

The indirect incentives include service and environmental factors that motivates agents in the system [12]. Take ride sharing vehicle-based multi-agent system as an example. The daily ride requests for taxis constitute such a service incentive. Although it is hard to reproduce such an indirect incentive, it is possible to analyze and utilize the indirect incentives to work with direct incentives for better systematic performance [13]. The major question is how to quantify the indirect incentives to make it comparable with the direct incentives.

\section{Simulation Platform}

For multi-agent systems with incentive schemes, due to the complicated interactions between agents and the environment, it is difficult to create an actual prototype and environment to evaluate the effectiveness. A simulation platform is a cheap but valid way in analyzing and testing for different collaborative algorithms, prior to real environmental implementations.

In addition, real world data is usually integrated in a simulation platform to better mimic agents' behaviors and status in real world scenarios. However, it is usually difficult to directly apply collected real data to a simulation platform for multi-agent systems with incentive schemes. This is because the incentives can continuously change the behaviors (e.g., direction to move) and statues (e.g., locations) of agents while the real-world data does not include any incentive information and thus the changed behavioral information [14], making it difficult to simulate the agents behavior onward using the real-world data. Therefore, the simulation platform should be able to produce new data to continuously emulate behaviors of agents. Existing simulators are inadequate for realistic analysis on multi-agent systems with incentive schemes, since they do not -

- Provide two different types of incentives (i.e. direct and indirect) and make them quantitatively comparable.

- Generate data to emulate behaviors of agents after they are incentivized.

We hereby present a physical knowledge aided multiagent simulation platform with incentive schemes. Physical knowledge is applied on real world data to extract historical features, based on which a generated dataset is produced to better accommodate simulation. The generated dataset still resembles the original dateset and expands as the real dataset grows by continuously tracking the newly generated behavior and status information of agents that are incentivized. As a result, the model learns to produce more accurate dataset to best take advantage of the given real data. In addition, two types of incentives (direct and indirect) are provided for different incentive scheme designs and physical knowledge is adopted to make these incentives quantitatively comparable and transformable. We examine the effectiveness of the platform with an example scenario of ride sharing vehicle based multi-agents systems.

The rest of the paper is organized as follows. Section 2 provides a brief description of various components and design of the framework. Example simulation and related discussion are given in Section 3. Section 4 discusses previous related work in this field. Finally, the paper ends with conclusion and future view in Section 5.

\section{SIMULATION FRAMEWORK}

The simulation framework takes two types of input: real data and customized data. The real data is used to shape the framework by providing the ground-truth data, while the customized data is used for testing our strategies. The latter can be either collected from environments different 


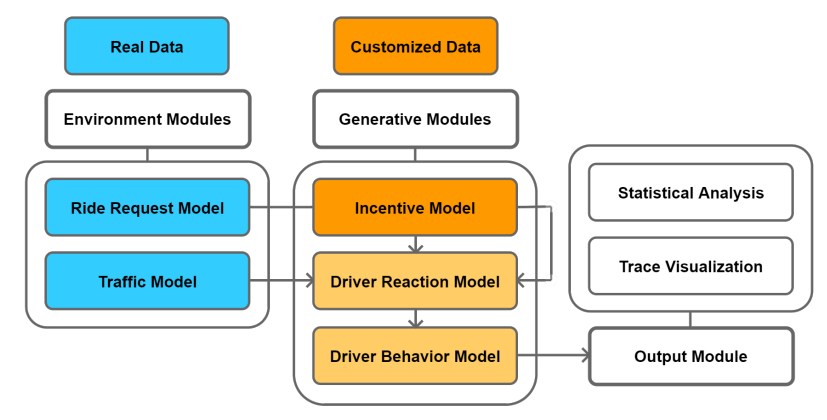

Figure 2: Simulation Framework

from those for the real data or generated from customized functions designed for each benchmark experiment run by our simulator. For instance, we may collect the data of moving obstacles of our agents which are not available in the same environment (e.g., city, period of times) where the real data are collected but can be added for testing the robustness and adaptability of our simulator. Our designed functions to incentivize agents also generate customized data that cannot be collected outside our simulator. These two types of input data may have conflicts with each other in certain circumstances. For instance, the moving behavior of the simulated agents will be affected when our incentives are imposed upon the agents. Thus the original real data set cannot be applied here any more. While we will elaborate how the conflicts are resolved in the later section, we emphasize here that the co-existence of the real data and customized data is necessary despite their conflicts. The real data, such as the background traffic environment and ride requests, are used to feed the environment modules to ensure the validity of the simulation framework. On the other hand, the customized data serve as a glue between the real world and the simulation platform, which need to be tested and can be improved over time.

\section{Environment Modules}

The environment modules consist of the traffic model and the ride request model. These models are environmental configurations that the whole simulation is based on. The traffic model uses the data of the number of vehicles in each location which can derive the speed, cost, and other information of agents that traverse each location. One can also import other data such as the road conditions and weather conditions to provide more diverse dynamics of each location that could affect the agents' behavior. The ride request model gives systematic request orders to each agent, which can be regarded as one type of indirect incentive to agents, as rationale agents generally move towards locations with more ride requests in order to improve their utilities if other factors are fixed. Both models in the environment modules are built on top of real data and can back up the validity of the simulator, however, do not simply copy the real data to feed into the agents' behavior model. More specifically, our environment modules include functions that pre-process the raw data, e.g., by pruning the samples that are affected by some identified extreme weather conditions and public events and thus impede the generality of the data. In addition, we are able to expand the data set using the extracted features so that the expanded data set can cover a longer period of system span and also a wider range of environment dynamics.

\section{Generative Modules}

The generative modules serve the central need to provide contexts for customized data. Similar as the usage of real data, the customized data may also be hard to fit directly into our simulator. To address this, we make the customized data compatible in the following way. If the customized data are generated outside our simulator and imported to enrich our environment characteristics, we first use similar functions to pre-process them as those used for the real data. Otherwise, the data are generated by the functions in our incentive model, driver reaction model, and driver behavior model. These models are able to automatically fill in the gaps between our generated customized data and the real data in an online fashion, e.g., by sampling real data from the historical samples recorded in the environment modules to reshape our generated data in order to satisfy the requirements imposed on the environment or agents. Specifically, the incentive model gives direct incentives, usually in the form of money, to agents to encourage an otherwise unlikely move. These incentives are one type of customized data; together with the data generated by the environment module, they are taken into account by the agents reaction model. Based on the incentives, deterministic policies for each agent are defined in our driver reaction model to simulate their choices in real time. The overall simulation will be performed under the driver behavior model where predictions regarding target agents can be made. In the following, we describe each model within the generative module.

Incentive Model. We generate real-time incentive signals for each agent so as to indirectly manipulate how agents move in our incentive model. We provide a flexible incentive model with parameters that can be adjusted such as the granularity and scale of the incentives, as well as the frequency to update incentives in the time horizon. These incentive signals can either be positive, like monetary bonus and credit points, or negative, such as alerts and punishments. In our major use case, we design a monetary-based incentive system that generates offers to rebalance agents. Along with 


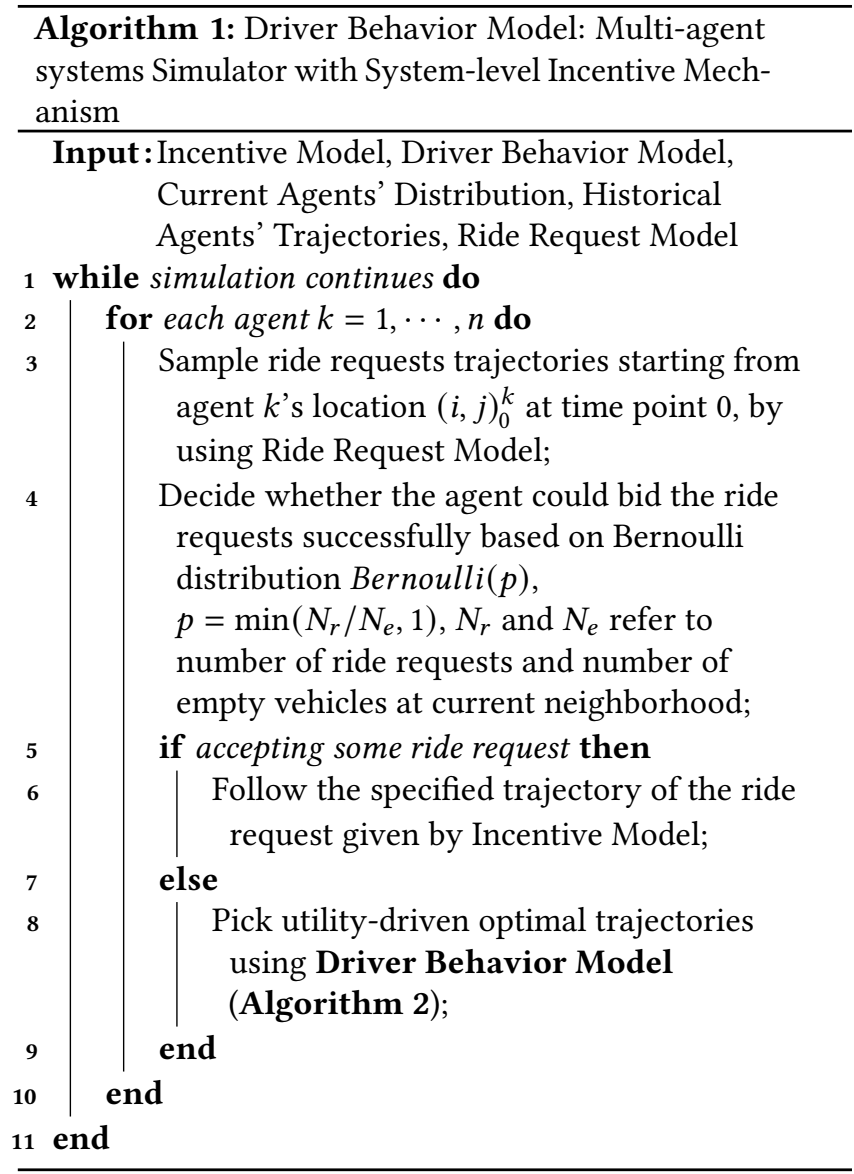

the previous ride request model, the incentive model creates environmental encouragements to lead agents towards the desired destinations of the system, e.g., to minimize the dissimilarity of the resulted distribution of agents and a certain target, minimize the total monetary cost, or increase the acceptance rate. In addition, by analyzing how agents interact with the given incentives and the simulation environment, the incentive model can adjust itself to achieve the system goal more effectively.

Driver Reaction Model. We define how agents react to the simulation environment in the driver reaction model. In our simulation platform, each agent determines its next move according to its own evaluation functions and various environmental factor models aforementioned: real-time traffic, ride requests, and generated incentives. The evaluation function is based on a probabilistic utility-driven strategy where the agent will choose the move that can achieve the highest estimated utility. In real world, agents may have limited knowledge of the environment or a restricted set of choices to move. Given these, we first generate a fixed set of candidate directions for each agent and assume that they are

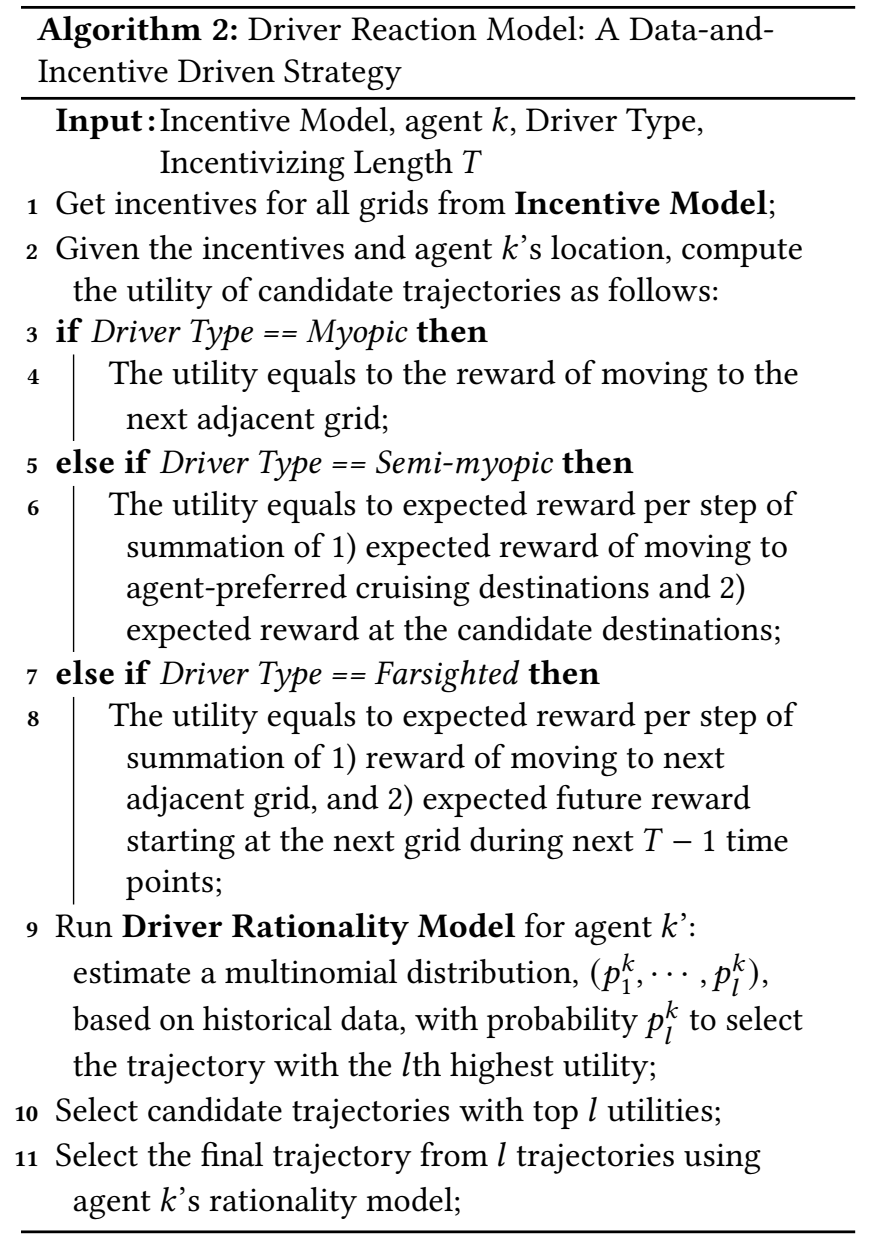

rationale in that they make decisions to maximize their utilities but can also have different preferences in shaping their utility functions based on their own estimations. Our implemented utility of each candidate direction is a function of the cost to move in that direction and the incentives including the ride requests and our generated monetary incentives. We construct three types of driver reactions to specify the utility function that we adopt: Myopic, Semi-myopic, and Farsighted reactions, in each of which the utility calculation is presented in our Algorithm 2. Myopic refers to that an agent only focuses on the utility during the forth-coming time point and ignore long-term reward. Semi-myopic refers to that an agent would consider the utility during a shortterm time period, for example, there are some neighborhoods which have higher ride request rates, semi-myopic agents are willing to sacrifice immediate utility to get more average utilities in the short-term future. Farsighted agents would estimate the utility of a long-term future until the end of incentivizing period. To capture the rationality and heterogeneity of agents, we construct a probabilistic strategy 
for each agent, which assigns the probability of moving in each direction according to the associated utility, so that the agents always tend to choose the moves with higher estimated utilities, but still have small probabilities to choose the moves with lower utilities, which is modeled by Driver Rationality Model shown in Algorithm 2. Such probabilistic utility-driven strategy simulates the interactions between the agents and the environment.

Driver Behavior Model. The behavior of each agent is simulated following the same form of reaction function described above. More specifically, in each time interval of our simulation, the agents (drivers of taxis, ride-sharing cars, etc.) in the entire simulated spatial area are either in the status of occupied (taking passengers) or idle. The occupied agents are not incentivized, and they always follow the trajectories of the taken ride requests, specified in the real data traces. For idle agents, their behavior is simulated by our behavior function which consists of two main steps: 1) the agent decides whether to take a ride request at the current location immediately, according to a random distribution determined by the total numbers of the ride requests and idle taxis at the corresponding location; 2) If the agent takes a ride request, it will then follow the corresponding trajectory specified by the request. Otherwise, the agent selects the next location to go according to the probabilistic utility-driven strategy defined in our driver reaction model. Whenever the probabilistic utility-driven strategy is applied to an agent, the corresponding monetary incentive for the selected next location can be collected. In our current implementation, the spatial area is split into discrete grids, each of which represents a location. These two steps together naturally simulate the behavior of a rationale agent, who chooses her next location with the largest utility with high probability. Since the main job of each agent is to take passengers, the first step of the behavior function is to randomly decide whether the agent takes a request before accepting our monetary incentives and deciding the next location. In this way, we simulate agents who always see their main jobs as the first priority, by regarding the ride requests as dominated incentives. One can also modify the behavior function of idle agents to be a single step of directly using the probabilistic utility-driven strategy, if the the utility of taking ride requests can also be modeled comparable to our monetary incentives.

\section{Output Modules}

To better understand the agents' behavior, the data traces of agents' movements and trajectories can be collected in real time. New statistical patterns in any simulation metrics can be extracted by analyzing the generated data. In addition, we also provide a function to visualize the data traces, which will be shown in Section 3.

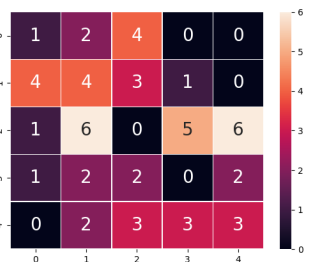

(a) ride requests

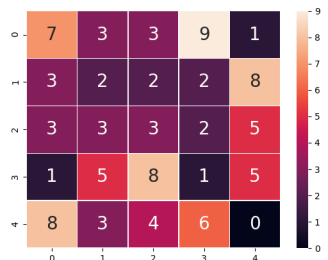

(b) incentive pricing
Figure 3: Distributions of ride requests and the pricing table

Essentially the incentives generated in our incentive model are used to induce agents to selectively deviate from their original trajectories so that the rebalanced agents can automatically fulfill the tasks designed by the simulator. For instance, the new distribution of the agents can be less crowded anywhere than the one without monetary incentives, if we generate the right incentives to feed agents.

\section{EXAMPLE SIMULATION SCENARIO AND DISCUSSION}

In this section, we provide example scenario simulation results to showcase the advantages of our simulator. Our simulator has the capacity to take into account both indirect and direct incentives, generate desired trajectories based on historical data, and incorporate different algorithms to incentivize agents. Our simulation utilizes the data traces of taxi vehicles in Beijing, China [15], and we show various simulation results on our extracted ride requests, generated incentives, and taxi distributions. We finally adopt the KLdivergence [16] as a performance metric to characterize the dissimilarity between the initial taxi distribution extracted from the data traces and a pre-determined target one that our platform tries to approach by using "right" incentives, and we show the KL-divergence comparison with different incentives used in our simulations. We leave the results on using DRL for learning the optimal incentives into our future work.

We run the simulation over 400 episodes and we repeat the simulation using the environmental data over the same day for each episode during simulation. We discard the period between 0 am to 8 am as few vehicles are active during that time of day. For the rest of the day, we split the time horizon into 2-minute time steps to keep track of vehicle movements. The simulator organizes real world location information into grid structures to coordinate agents and incentives. The simulation initializes the locations of 100 agents randomly over the map during each episode, and then agents move according to the probabilistic utility-driven strategy described in Section 2. In addition, for this example scenario, we adopt the Myopic Behavioral Model in Algorithm 1 to generate the 


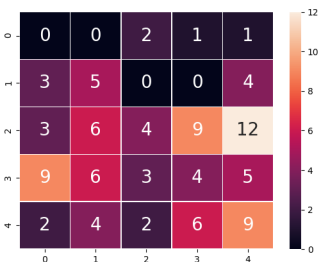

(a) without incentives

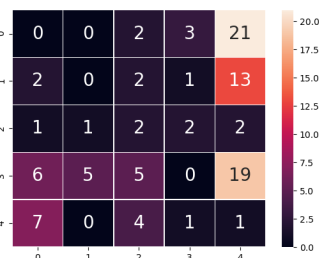

(b) with uniform incentives

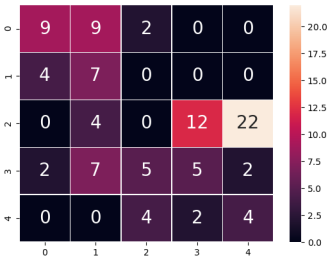

(c) with random incentives

Figure 4: Distributions of drivers with uniform, random incentives and without direct incentives

agents' reactions to the incentives and generate the figures shown in the following.

When a direct incentive model is present, in addition to the normal ride request orders, agents also receive pricing tables as direct incentives. The following figures show the example distribution maps of the systematic ride requests and pricing tables assigned by incentive model in a given time step, where each number in the grid represents the number of ride requests and incentive levels (a higher number means a higher incentive), respectively. They serve as instantaneous indirect and direct incentives to mobilize agents and can affect the distribution of the agents in one or more future time steps.

Agents have routes to follow when they accept ride request orders, but they may accept our incentives based on the posted price map and thus deviate from their original routes or change the destinations. The trajectories of the deviated taxis do not follow the original data traces any more and need to be simulated by our driver behavior model. In response to that, we device a generated path between its current position and the destination.

In figure 5, we demonstrate how agents move when real data cannot be directly applied in such a hybrid-incentivized environment. The trajectory is generated by our simulator based on the historical values of the same end-to-end paths. We made the generated paths to be with as few turns as possible because we assume normal drivers are more comfortable with going in a straight direction. According to our dataset, most drivers do not take more than 2 turns on their trajectories. They show preference for straightforward paths

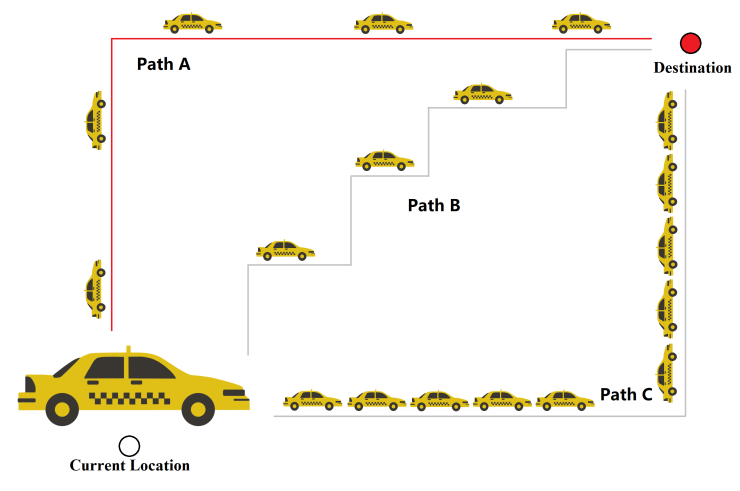

Figure 5: Taxi trajectories showcase

like Path A. Curly trajectories like Path B would not be considered as the best route to take in this case. In addition, real time traffic also contributes to the path generation. The agent will prefer Path A with less traffic over Path $\mathrm{C}$ that is overcrowded with other vehicles. The generated trajectories would cost the driver relatively less time and effort, and we believe paths like Path A best resemble paths drivers would take in real world.

Figure 6 illustrates the results of an example scenario when different incentives are assigned to the model. We use the $\mathrm{KL}$ divergence between the distributions of agents as an indicator for algorithm potency. The KL divergence is a scalar value indicating the dissimilarity between two distributions. The lower the value for KL divergence, the more similar the distributions. Our target distribution consists of a uniform distributed center and empty edges. In the example, we compare the simulation results with and without an incentive input to the environment. As shown in figure 6, when fed with incentives that value more in the central grids, the KL divergence are generally smaller than the one without incentives. The vehicle distribution is thus closer to the target distribution when a positive incentive is given. Incentives proved effective for our simulation model in monitoring agent distributions.

\section{RELATED WORK}

In this section, we introduce several related works that aim to solve similar problems with inspiring solutions. A number of multi-agent platforms have been developed for simulation.

The GAMA platform provides an agent-based modelling and simulation development environment[17]. It allows professionals with inadequate knowledge about computer science concepts to build simulations. It has been applied in various fields including transportation and urban planning. 


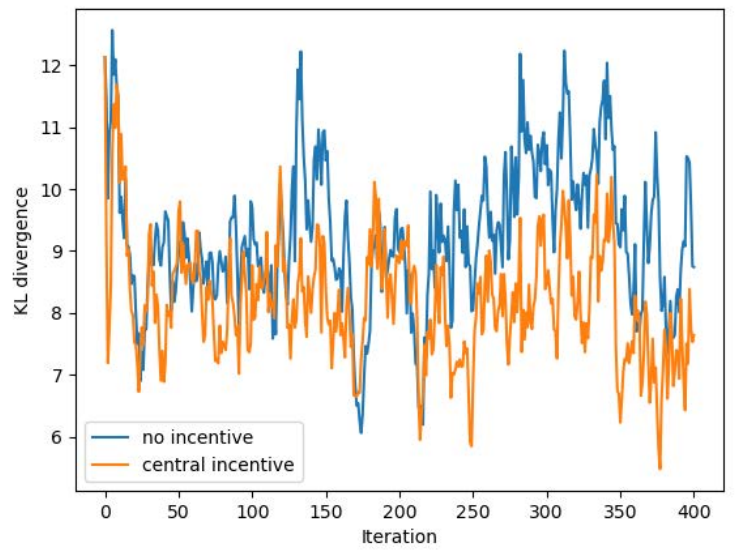

Figure 6: KL-divergence under different incentives

Although the GAMA platform appeals to a general multiagent setting, it does not take into account the specific environmental data adaptation and does not particularize the effect of different incentives.

At UC Berkeley, the Flow platform provides a modular learning framework for traffic[18]. It incorporates model-free reinforcement learning methods to address the complexity of traffic dynamics. The platform focuses on traffic autonomy and its control law achieved great performance. Due to its model-free nature, however, precise tuning of incentives is not easy.

A similar simulation framework also appears for indoor fire monitoring[19]. The group used controlled sensors as agents to respond to emergency situations. Both cyber and physical components work together to produce a realistic simulation. The framework provides a successful example for simulators, but the subject covers a less variant environment.

The simulation framework presented in this paper combines a multi-agent platform with two types of incentives. In addition, the simulator generates data from real data to accommodate inapplicable situations. These novel concepts should contribute to future related frameworks.

\section{CONCLUSION}

This paper presents a physical knowledge aided multi-agent simulation platform with both direct and indirect incentives, which are quantitatively comparable and transformable. Physical knowledge is applied on real world data to extract historical features, based on which a generated dataset is produced to better accommodate simulation. The generated dataset still follows a physical knowledge background and expands as the real dataset grows. We examine the effectiveness of the platform with the example scenario of ride sharing vehicle based multi-agents systems.

\section{REFERENCES}

[1] Jacques Ferber and Gerhard Weiss. Multi-agent systems: an introduction to distributed artificial intelligence, volume 1. Addison-Wesley Reading, 1999.

[2] Xinlei Chen, Carlos Ruiz, Sihan Zeng, Liyao Gao, Aveek Purohit, Stefano Carpin, and Pei Zhang. H-drunkwalk: Collaborative and adaptive navigation $<$ ? brk? > for heterogeneous mav swarm. ACM Transactions on Sensor Networks (TOSN), 16(2):1-27, 2020.

[3] Peter Stone and Manuela Veloso. Multiagent systems: A survey from a machine learning perspective. Autonomous Robots, 8(3):345-383, 2000.

[4] Guang Wang, Yongfeng Zhang, Zhihan Fang, Shuai Wang, and Desheng Zhang. Faircharge: A data-driven fairness-aware charging recommendation system for large-scale electric taxi fleets. 2020.

[5] Susu Xu, Xinlei Chen, Xidong Pi, Carlee Joe-Wong, Pei Zhang, and Hae Young Noh. ilocus: Incentivizing vehicle mobility to optimize sensing distribution in crowd sensing. IEEE Transactions on Mobile Computing, 2019.

[6] Xinlei Chen, Aveek Purohit, Carlos Ruiz Dominguez, Stefano Carpin, and Pei Zhang. Drunkwalk: Collaborative and adaptive planning for navigation of micro-aerial sensor swarms. In Proceedings of the 13th ACM Conference on Embedded Networked Sensor Systems, pages 295-308, 2015.

[7] Jing Xie and Chen-Ching Liu. Multi-agent systems and their applications. Journal of International Council on Electrical Engineering, 7, 2017.

[8] Shuai Wang, Tian He, Desheng Zhang, Yunhuai Liu, and Sang H. Son. Towards efficient sharing: A usage balancing mechanism for bike sharing systems. In The World Wide Web Conference, pages 2011-2021, 2019.

[9] Xinlei Chen, Xiangxiang Xu, Xinyu Liu, Hae Young Noh, Lin Zhang, and Pei Zhang. Hap: Fine-grained dynamic air pollution map reconstruction by hybrid adaptive particle filter. In Proceedings of the 14th ACM Conference on Embedded Network Sensor Systems CD-ROM, pages 336-337, 2016

[10] Songyuan Li, Shibo He, Shuai Wang, Tian He, and Jiming Chen. Datadriven battery-lifetime-aware scheduling for electric bus fleets. Proceedings of the ACM on Interactive, Mobile, Wearable and Ubiquitous Technologies, 3(4):1-22, 2019.

[11] Zhihan Fang, Yu Yang, Shuai Wang, Boyang Fu, Zixing Song, Fan Zhang, and Desheng Zhang. Mac: Measuring the impacts of anomalies on travel time of multiple transportation systems. Proceedings of the ACM on Interactive, Mobile, Wearable and Ubiquitous Technologies, 3(2):1-24, 2019.

[12] Xinlei Chen, Susu Xu, Haohao Fu, Carlee Joe-Wong, Lin Zhang, Hae Young Noh, and Pei Zhang. Asc: Actuation system for city-wide crowdsensing with ride-sharing vehicular platform. In Proceedings of the Fourth Workshop on International Science of Smart City Operations and Platforms Engineering, pages 19-24, 2019.

[13] Susu Xu, Xinlei Chen, Xidong Pi, Carlee Joe-Wong, Pei Zhang, and Hae Young Noh. Vehicle dispatching for sensing coverage optimization in mobile crowdsensing systems. In 2019 18th ACM/IEEE International Conference on Information Processing in Sensor Networks (IPSN), pages 311-312. IEEE, 2019.

[14] Xinlei Chen, Susu Xu, Jun Han, Haohao Fu, Xidong Pi, Carlee JoeWong, Yong Li, Lin Zhang, Hae Young Noh, and Pei Zhang. Pas: Prediction-based actuation system for city-scale ridesharing vehicular mobile crowdsensing. IEEE Internet of Things fournal, 7(5):3719-3734, 2020 . 
[15] Beijing taxi data. http://sensor.ee.tsinghua.edu.cn/datasets.html, 2015.

[16] Kullback-leibler divergence. https://en.wikipedia.org/wiki/Kullback\% E2\%80\%93Leibler_divergence, 2020.

[17] Patrick Taillandier, Benoit Gaudou, Arnaud Grignard, Quang-Nghi Huynh, Nicolas Marilleau nad Philippe Caillou, Damien Philippon, and Alexis Drogoul. Building, composing and experimenting complex spatial models with the gama platform. Geoinformatica, 23:299-322,
2019.

[18] Cathy Wu, Aboudy Kreidieh, Kanaad Parvate, Eugene Vinitsky, and Alexandre M. Bayen. Flow: Architecture and benchmarking for reinforcement learning in traffic control. CoRR, abs/1710.05465, 2017.

[19] A. Purohit and P. Zhang. Controlled-mobile sensing simulator for indoor fire monitoring. In 2011 7th International Wireless Communications and Mobile Computing Conference, pages 1124-1129, 2011. 